\title{
Agentes externos, Ciudades Educadoras e Inclusión. Ejes del cambio en las aulas.
}

\author{
Ana Amaro Agudo \\ Universidad de Granada \\ anaamaro@eulainmaculada.com \\ Diego NaVArro Mateu \\ Universidad Católica de Valencia \\ diego.navarro@ucv.es
}

\section{Resumen}

En las últimas décadas se ha producido un cambio en la concepción de la educación, diferentes organismos internacionales han reconocido que la educación inclusiva es la mejor manera de brindar una educación para todos. En este artículo recogemos las características del proyecto iniciado sobre voluntariado, Ciudad Educadora e inclusión como ejes de un cambio posible en nuestras aulas.

Palabras Clave: Agentes externos, voluntariado, Ciudad Educadora, Educación Inclusiva.

\section{External agents, educational cities and inclusion. Axes of change in the classrooms.}

\begin{abstract}
In recent decades there has been a change in the conception of education, several international organization shave recognized that inclusive education is the best way to provide education for all. In this article we collect the features of the project started about volunteering, educational city and inclusion as axes of a possible change in our classrooms.
\end{abstract}

Key Words: External agents, volunteering, educational city, inclusive education.

\section{Referencia normalizada:}

Armaro Agudo, A. y Navarro Mateu, D. (2013) Agentes externos, Ciudades Educadoras e Inclusión. Ejes del cambio en las aulas. Historia y Comunicación Social. Vol. 18. N $^{\circ}$ Especial Diciembre. Págs. 449-460.

Sumario: 1. Introducción. 2. Metodología empleada. 3. Legislación y principales aportaciones de organismos. 4. Necesidad de transformar la escuela. 5. Ciudad educadora como estructura de apoyo al cambio. 6. Voluntariado educativo. 7. Proyecto de colaboración Ciudad Educadora-voluntariado educativo. 7.1. Objetivo general del proyecto. 7.2. Objetivos específicos. 7.3. Fases del proyecto. 8. Conclusiones. 


\section{Introducción}

En las últimas décadas, distintos organismos internacionales, a través de foros y conferencias como la "Conferencia Mundial sobre Educación para Todos: Atendiendo Necesidades Básicas": Joimen (Tailandia),1990, la "Conferencia Mundial sobre NEE" Salamanca (España), 1994, o el "Foro Mundial sobre educación" (Senegal), 2000, han ido reconociendo que la Educación Inclusiva es la mejor manera de brindar una educación para todos y todas, como modelo hacia el que deben dirigirse todos los sistemas educativos.

La profunda transformación social acaecida en las últimas décadas, en las que el derecho a una educación sin distinciones ni etiquetajes va abriéndose paso obliga moral y éticamente al profesorado, y a los especialistas en educación, a ayudar y dar un giro al concepto de educación que llevamos arrastrando desde la sociedad industrial. A lo largo del artículo realizamos una fundamentación teórica sobre los conceptos clave en los que se enmarca nuestro estudio. Con el objeto de afianzar y consolidar nuestro proyecto.

El proyecto que aquí presentamos tiene como finalidad realizar una propuesta de intervención en centros escolares financiados con fondos públicos, con el objetivo de mejorar la cultura inclusiva del centro, que permita una optimización de los indicadores que se establezcan, mediante el desarrollo de un programa de voluntariado educativo, desarrollado en colaboración con las ciudades a las que pertenezca el centro escolar.

\section{Metodología empleada}

Consideramos necesario articular una metodología de investigación-acción para nuestro proyecto y, debido a la complejidad de la realidad educativa, la misma tendrá un carácter plurimetodológico.

La investigación-acción es una forma de investigación que permite vincular el estudio de los problemas, en un contexto determinado, con programas de acción social, de manera que se logren de forma simultánea conocimientos y cambios sociales.

Es un instrumento potente e inmediato para la transformación de la enseñanza, cuyos elementos son articulados en la práctica, de manera que la investigación es el potencial del educando: su preocupación, su colaboración y su perfeccionamiento; mientras que la acción es la actividad teórica para desarrollar el potencial del educando.

Además, constituye un recurso científico dirigido a estudiar, controlar y alcanzar las modificaciones deseadas en el entorno social de aplicación. Se centra en la posibilidad de aplicar categorías científicas para la comprensión y mejoramiento de los procesos de transformación, partiendo del trabajo colaborativo de los propios 
sujetos implicados. Por ello, constituye una vía excelente para eliminar la dicotomía teoría-práctica y sujeto-objeto de la investigación, característicos de la investigación tradicional en al campo de la educación.

Esta metodología cualitativa se adapta mejor a nuestro estudio educativo, aunque debe existir una complementariedad con técnicas cuantitativas. Para ello utilizaremos un pretest, antes de comenzar la implementación de nuestro proyecto, seguido de un postest, al concluir el período de aplicación del mismo. El objetivo de dotar a nuestra investigación de este carácter plurimetodológico es proceder, a continuación, a una triangulación de datos, con lo que aumentemos la confiabilidad de este estudio. Ello le dotará de mayor validez interna, así como de fiabilidad al comparar los resultados obtenidos con los distintos métodos.

\section{Legislación y principales aportaciones de organismos.}

Durante muchos siglos, las personas discapacitadas eran rechazadas y excluidas de la sociedad. La mirada al pasado nos confirma que el concepto de Educación Especial ha sufrido fuertes transformaciones a lo largo de los años. Ejemplo de ello son los momentos clave que señalamos a continuación.

El año1948, año de la Declaración de los Derechos Humanos del Hombre por Naciones Unidas, marca un antes y un después en torno a la defensa de los derechos y libertades individuales. La Declaración es un hito indiscutible en la historia de la humanidad, ya que supone el primer reconocimiento universal de los derechos básicos y las libertades fundamentales.

El Informe Warnock, publicado en el Reino Unido en 1978 y realizado por una comisión de expertos presidida por Mary Warnock, contribuyó a modificar radicalmente el concepto de Educación Especial. Estableció una nueva denominación para aquellos alumnos que presentan, a lo largo de su escolarización, problemas en sus aprendizajes: alumnos con necesidades educativas especiales. Esta nueva denominación no fue, solamente, un cambio terminológico, supuso el impulso a una nueva concepción, que evitaba el lenguaje de la deficiencia, y situaba en la propia escuela una buena parte de la responsabilidad sobre el progreso de los alumnos.

Casi un cuarto de siglo más tarde, en junio de 1994, se celebró en España la que se ha dado en llamar "Declaración de Salamanca", conferencia organizada por el Gobierno Español en colaboración con la UNESCO (UNESCO, 2004). En ella se congregaron altos cargos de la educación, administradores, responsables de las políticas educativas, técnicos especialistas y representantes de Naciones Unidas, con el fin de promover el objetivo de "La Educación para Todos".

La citada Declaración y el Marco de Acción de Salamanca para las Necesidades Educativas Especiales, que de ella se derivó, fueron adoptados por unanimidad por los representantes de 92 gobiernos y 25 organizaciones internacionales, y sin ninguna 
duda se ha convertido en un importante referente para la innovación pedagógica y para las políticas educativas.

De forma paralela, la legislación estatal, ha ido recogiendo este progresivo reconocimiento de derechos, concretándolos en las diferentes Administraciones educativas que, a lo largo de estos años, han dado un giro vertiginoso hacia la educación sin exclusiones y hacia la necesidad de una educación más inclusiva.

Como ejemplo de ello:

Ley Orgánica de Ordenación General del Sistema Educativo (L.O.G.S.E., 1990). Esta Ley introduce por primera vez el concepto de necesidades educativas especiales, que se recoge en el artículo 36:

"El sistema educativo dispondrá de los recursos necesarios para que los alumnos con necesidades educativas especiales, temporales o permanentes, puedan alcanzar, dentro de un mismo sistema, los objetivos establecidos con carácter general para todos los alumnos" (artículo 36.1).

Ley Orgánica de Calidad de la Educación (L.O.C.E., 2002). Esta Ley restringe el concepto de necesidad educativa especial e introduce un concepto más amplio: el de "necesidad educativa específica",

Ley Orgánica de Educación (L.O.E., 2006). Cambia el término de "necesidad educativa específica" por el de "necesidad específica de apoyo educativo".

"Corresponde a las Administraciones educativas asegurar los recursos necesarios para que los alumnos y alumnas que requieran una atención educativa diferente a la ordinaria, por presentar necesidades educativas especiales, por dificultades específicas de aprendizaje, por sus altas capacidades intelectuales, por haberse incorporado tarde al sistema educativo, o por condiciones personales o de historia escolar, puedan alcanzar el máximo desarrollo posible de sus capacidades personales, y, en todo caso, los objetivos establecidos con carácter general para todo el alumnado" (L.O.E., art. 71.2). "[...] La atención integral del alumnado con necesidad específica de apoyo educativo se regirá por los principios de normalización e inclusión" (L.O.E., art. 71.3).

\section{Necesidad de transformar la escuela.}

Este proceso abre una mirada esperanzadora al cambio educativo tan esperado en nuestras aulas, y a la confianza en el camino que aún queda por recorrer. Los cambios educativos se están produciendo y hemos visto su evolución. La escuela ha iniciado un camino, en el que de forma progresiva la lleva a reformarse y reformular sus estrategias, así como sus herramientas pedagógicas, para poder dar respuesta positiva y real a la diversidad de su alumnado, contemplando la diversidad individual no como un problema, sino como una oportunidad de enriquecer los aprendizajes. 
Vamos a destacar dos conceptos que consideramos nucleares, para facilitar y contribuir al desarrollo de una educación inclusiva: el concepto de Cultura Escolar, en concreto refiriéndonos a las dificultades a las que se enfrentan los alumnos en su interacción escolar y el concepto de comunidad educativa, concepto necesario para el desarrollo de la educación inclusiva.

Consideramos que la Cultura Escolar, entendida a partir del conjunto de creencias y convicciones que tienen los miembros de la comunidad educativa, en relación a la enseñanza y al aprendizaje del alumnado, así como con el funcionamiento del centro, no ha entendido ni asimilado un concepto nuclear, como es el de "barreras para el aprendizaje y a la participación" (Ainscow, M., 2001; Booth, T. y Ainscow, M., 2004; Stainback, S. y Stainback, W., 1999). Este concepto ha enfatizado y puesto de manifiesto la importancia del contexto en la determinación del grado de dificultades resultantes, para evitar que se siga atribuyendo al alumnado la responsabilidad de su fracaso, excluyendo en gran medida, de esta atribución, los aspectos organizativos, metodológicos y actitudinales inherentes al centro escolar, con lo que éste no es capaz de identificar e impulsar aquellos cambios internos que permitan una mejora en la participación de los alumnos.

Otro concepto nuclear en el desarrollo de una Educación Inclusiva, es el de Comunidad Educativa. El compromiso de la misma debe ser garantizar los derechos de los alumnos, movilizando, simultáneamente, tanto la captación de recursos externos de carácter administrativo, como los recursos pertenecientes a la comunidad educativa, formada tal y como dice la LOE por:...

"la responsabilidad del éxito escolar de todo el alumnado no sólo recae sobre el alumnado individualmente considerado, sino también sobre sus familias, el profesorado, los centros docentes, las Administraciones educativas y, en última instancia, sobre la sociedad en su conjunto, responsable última de la calidad del sistema educativo". (LEY ORGÁNICA 2/2006, de 3 de mayo, de Educación.PP.17159).

Todo lo anteriormente expuesto sobre cultura escolar y comunidad educativa es sin duda uno de los puntos clave para facilitar y promover el desarrollo y expansión de los círculos de empatía, término acuñado por Peter Singer en 1981 (Singer,1981), para definir un proceso mediante el cual cada vez sentimos empatía hacia más personas, permitiendo y facilitando el desarrollo de actitudes y conductas más solidarias que permiten una mejor desarrollo de entornos cordiales para todos.

\section{Ciudad educadora como estructura de apoyo al cambio.}

La propuesta que nosotros realizamos de enlazar escuela con entorno surge del concepto de Ciudad Educadora. Vamos a pasar a explicarla como punto previo a la descripción del Programa de Voluntariado Educativo, con el que pretendemos aumentar los recursos humanos del centro escolar, contribuyendo, de esta forma, a garantizar la adecuada atención educativa a todos los miembros de la misma. 
Partimos del concepto "Ciudad Educadora", que es el primer eje en que se sustenta este trabajo. Este concepto ofrece un contexto nuevo donde convergen la educación formal, la no formal y la informal, la cual, a través de sus proyectos, y del cumplimiento de la Carta de Ciudades Educadoras, se convierten en un eje, pilar y apoyo para cumplir con la demanda de apertura al entorno de todos los alumnos. El proyecto de investigación que planteamos, propone impulsar el trabajo de las Ciudades Educadoras como una estrategia de apoyo externo a los centros escolares. Dentro de la Ciudad Educadora se enlazan los tres tipos de educación, formal, no formal e informal como aparece recogido en (Gráfico 1).

Gráfico 1: Educ. formal, no formal e informal. Amaro (2002)

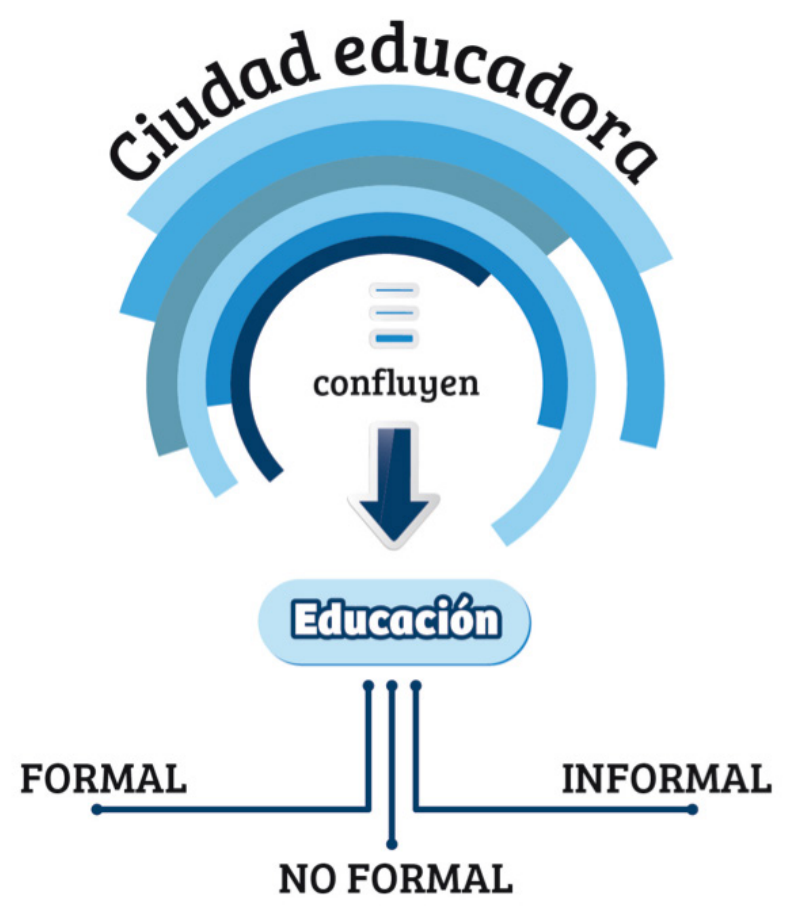

"La ciudad educadora es aquella que, consciente del papel fundamental de las ciudades en la educación de las personas, manifiesta su voluntad de incidir en ellas positivamente" La Ciudad Educadora" AAVV- Ajuntament de Barcelona, 1990.

\section{Voluntariado educativo}

El segundo eje en el que se sustenta el proyecto es el Voluntariado Educativo. Este voluntariado, externo a la escuela, es utilizado como medio de facilitar e incrementar la vinculación de la escuela con la comunidad a la que pretende servir, y de esa forma, 
incrementar las sinergias educativas de todos los miembros de la comunidad, se trate de agentes internos o externos.

La ciudad debe recuperar a las escuelas y las escuelas a las ciudades, encontrándose unos con otros en la tarea de conseguir el pleno derecho a la educación de todos sus ciudadanos, especialmente los más vulnerables. Esta relación ya está siendo llevada a la práctica en numerosas experiencias. Podemos observar cómo el concepto de voluntariado se ha hecho presente en grandes áreas asistenciales de nuestra sociedad, en la salud, la promoción social, el ámbito cultural, sin embargo, en el ámbito educativo todavía no existe una cultura de colaboración desde el voluntariado, salvada la excepción del voluntariado educativo llevado a cabo en las "Comunidades de Aprendizaje". Las únicas referencias encontradas en relación al desarrollo y uso del voluntariado en el interior de los centros escolares en el Estado español, hacen, todas ellas, referencia a las Comunidades de Aprendizaje. Las Comunidades de Aprendizaje son un modelo educativo que tiene como finalidad impulsar la transformación social y educativa de los centros educativos, con el objetivo de contribuir a la superación del fracaso escolar y a mejorar la convivencia. Para ello, y entre otras prácticas educativas, crean grupos heterogéneos dentro del aula en los que participan profesorado y voluntariado. En estos grupos interactivos (AUBERT y GARCÍA, 2001, citado en Valls, R. y Munté, A., 2010), afirma la persona adulta sirve de referente y dinamiza las interacciones en el grupo, promoviendo la solidaridad entre las y los estudiantes, con el objetivo de que todas y todos resuelvan con éxito las actividades.

Los voluntarios son personas de la comunidad (familiares, exalumnos/as, personas del barrio, miembros de asociaciones del barrio, estudiantes en prácticas, otros profesionales vinculados al barrio y a la comunidad...), que con su participación y compromiso en el quehacer diario de la escuela, aumentan el número de interacciones que viven los alumnos. Comunidades de aprendizaje se está expandiendo entre diferentes centros educativos de España, alcanzando ya cerca de una centena.

\section{Proyecto de colaboración ciudad educadora-voluntariado educativo.}

Desarrollada toda la fundamentación teórica de nuestro trabajo, pasamos a exponer el plan de provisión de agentes externos, como apoyo a la inclusión.

\subsection{Objetivo general del proyecto}

Intervenir en centros escolares financiados con fondos públicos, con la finalidad de generar una cultura inclusiva que contribuya a mejorar los entornos educativos, mediante la creación e implementación de un Programa de Voluntariado Educativo, en colaboración con las autoridades municipales en materia educativa. 


\subsection{Objetivos específicos}

Crear un espacio cooperativo entre diferentes instituciones (Autonómicas, Entidades Locales, movimiento asociativo...) para dar respuesta, de forma integral, a las necesidades del espacio educativo.

Crear una medida educativa de atención al alumnado: voluntariado educativo, capaz de operativizar, significativamente, las necesidades específicas de apoyo educativo.

Proporcionar la formación necesaria al voluntariado para atender las necesidades específicas de los centros, en las actividades de apoyo escolar.

Diseñar un programa funcional de intervención de los voluntarios en cada centro.

Evaluar los resultados educativos del centro con el objeto de establecer el alcance de la investigación.

Realizar el pilotaje de la intervención del voluntariado educativo en los centros.

Evaluar y transferir los resultados de la primera fase de la investigación para la puesta en marcha de la siguiente.

Facilitar la adaptación de materiales que permitan su difusión y transferencia a otras comunidades escolares

\subsection{Fases del proyecto}

A continuación vamos a exponer las diferentes fases del proyecto.

$1^{\text {a }}$ Fase: Consideramos que el proceso debe dar comienzo mediante una reunión entre los responsables educativos a nivel municipal, y los responsables de las comunidades educativas, en concreto los Consejos Escolares Municipales, regulados por la Ley Orgánica 8/1985 del Derecho a la Educación (LODE).Una vez presentada la propuesta de colaboración al Consejo Escolar Municipal, por parte de la Administración Local, son los diferentes centros los que deciden su participación o no en el Proyecto, una vez se les ha presentado el mismo.

Una vez deciden su participación, se debe dar respuesta a los siguientes conceptos: definición de Voluntario Educativo, perfil del mismo, tareas a realizar, descripción y proceso para la adscripción del voluntario a una situación educativa, detección de las necesidades de apoyo educativo no adecuadamente satisfechas, determinación de las metodologías educativas ajustadas a la nueva situación, así como determinación de los indicadores de rendimiento y percepción que se establezcan para la evaluación del programa.

$2^{\mathrm{a}}$ Fase: Implementación del programa: En esta fase se procede a la selección y formación del voluntariado. Los voluntarios reciben una formación básica por parte de las Autoridades Locales, y una formación específica gestionada por el propio 
centro. A continuación, se comienza con la asignación de los voluntarios a las diferentes situaciones educativas.

$3^{\text {a }}$ Fase: Evaluación del programa: Una vez implementado el programa, se procede a la recogida de datos en referencia a los indicadores establecidos, se realiza una puesta en común de los resultados, y se inicia la valoración global del programa en cada centro escolar. Posteriormente, se realizará una evaluación global, desde la perspectiva de la ciudad, para poder recoger las conclusiones y proceder a la elaboración de propuestas de continuidad, incorporando los cambios que se consideren oportunos.

$4^{\mathrm{a}}$ Fase: Transferencia de resultados y del proyecto: La idea del proyecto es llegar a demostrar la idoneidad del proyecto, de forma que pueda ser transferido a otras entidades locales, para ser desarrollado e impulsado en sus centros escolares, como una potente herramienta para facilitar la inclusión efectiva de todos sus ciudadanos en edad escolar, especialmente los más vulnerables.

\section{Conclusiones}

Debemos ser capaces de romper una inercia social, que acepta la presencia del voluntariado en gran parte de sus dimensiones, pero que, sin embargo, y aún a pesar de los limitados resultados que estamos obteniendo en la educación, no acepta la presencia de los voluntarios dentro del contexto escolar, dentro de las aulas.

Consideramos necesario incorporar y corresponsabilizar a la ciudadanía en la educación de sus ciudadanos, favoreciendo de esa forma la responsabilidad sobre ella. La ciudadanía activa implica una nueva cultura de la acción pública, y otorga una verdadera autonomía y responsabilidad a los ciudadanos.

Las ciudades, a través de sus competencias educativas, deben implicarse con sus comunidades educativas, creando estructuras interinstitucionales, que permitan dar una mejor cobertura al derecho a la educación de todos los ciudadanos, especialmente de los más vulnerables.

La presencia de agentes externos, consideramos que puede facilitar el desarrollo de una cultura inclusiva en las comunidades escolares. Va a obligar a los miembros de la comunidad educativa a reconsiderar sus propuestas educativas, identificando qué situaciones educativas no ofrecen la necesaria oferta de actividades para todos sus alumnos, de forma que, al corregir esto, todos ellos puedan participar activa, significativa y satisfactoriamente del currículum escolar.

Es lógico pensar que todo esto va a requerir un elevado compromiso de todos los agentes implicado, que sin duda, va a verse facilitado por esta colaboración entre la Comunidad Educativa y la Ciudad Educadora. 
Las hipótesis de partida que nos lleva a esta propuesta, es que los beneficios del programa de Ciudades Educadoras y Voluntariado Educativo va a suponer una potente herramienta de intervención e implicación social que, sin duda, beneficiará a todos los que participen de ella. En relación al alumnado, van a ver incrementada la calidad y cantidad de interacciones educativas, una mejor atención, y una presencia de modelos sociales pertenecientes a la comunidad. A los profesores, les va a permitir articular propuestas metodológicas al disponer de ayuda adicional, y enriquecerse de la presencia del voluntario, de sus habilidades e intereses. Los voluntarios van a poder contribuir al desarrollo de su comunidad, y a sentirse partícipes de un proyecto de transformación personal y social, por medio de su participación en los entornos escolares. A la comunidad educativa en general, esta colaboración le va a permitir un mejor logro de sus fines, y una mayor apertura al entorno del que forman parte.

\section{Referencias bibliográficas}

\section{Libros}

AINSCOW, M. (2001).Desarrollo de escuelas inclusivas. Madrid: Narcea

AMARO AGUDO, A. (2002) El movimiento de Ciudades Educadoras. Una investigación evaluativa. Tesis Doctoral. Granada: Universidad de Granada.

AYUNTAMIENTO DE BARCELONA. La Ciudad Educadora. AAVV- Ajuntament de Barcelona, 1990.

BOOTH, T; AINSCOW, M. (2004) Índice de inclusión: Desarrollando el aprendizaje y la participación en las escuelas. Santiago, Chile, OREALC/UNESCO, 2004. $117 \mathrm{p}$. (Versión original en inglés Booth T.;Ainscow, M. Centre for Studies on Inclusive Education (CSIE), Bristol, UK 2000.

DEBESSE, M y MIALARET, G. (1986). Formación continua y educación permanente. Barcelona: Editorial OIKOS-TAU, S.A.

FAURE, E y otros (1973). Aprender a Ser. Madrid: Alianza Universidad-UNESCO. Segunda edición.

STAINBACK, S. Y STAINBACK, W. [ed.] (1999). Aulas inclusivas. Madrid: Narcea. SINGER, PETER. (1981). The Expanding Circle: Ethics and Sociobiology, Farrar, Straus and Giroux, New York.

UNESCO (2005). Guidelines for inclusion Ensuring Acces to education for All. París: UNESCO.

UNESCO - (1994). Informe final: "Conferencia Mundial sobre Necesidades Educativas Especiales: Acceso y Calidad, Salamanca, España, 7-10 de junio de 1994”, "Declaración de Salamanca", conferencia organizada por el Gobierno español en cooperación con la UNESCO.

UNESCO (1990). Conferencia Mundial de Educación para Todos, Jontiem, Tailandia. París: UNESCO. 
UNESCO (1995). Las necesidades educativas en el aula. Conjunto de materiales para la formación de profesores. París: UNESCO.

UNESCO (2000). Foro Mundial de Educación para Todos, Dakar, Senegal. París: UNESCO.

UNESCO (2005). Guidelines for inclusion Ensuring Acces to education for All. París: UNESCO.

VERDUGO, M.A.; RODRÍGUEZ-AGUILELLA, A.; SARTO MARTÍN, P.; CALVO ÁLVAREZ, I. y SANTAMARÍA DOMÍNGUEZ, M. (2009). Situación Actual de la Inclusión Educativa en España. Informe de Investigación. Confederación Española de Organizaciones en favor de las Personas con Discapacidad Intelectual (FEAPS) e Instituto de Integración en la Comunidad. Universidad de Salamanca (INICO).

WARNOCK, H:M. "Special Educational Needs. Report of the Committee of Enquiry into the Education of the Handicapped Children and Young People", HMSO, Londres, 1978.Education.

Artículos en publicaciones web

TRILLA BERNET, J. y NOVELLA CAMARA, A. (2011): Educación y participación social de la infancia, en Revista Iberoamericana de Educación, $\mathrm{n}^{\mathrm{o}}$ 26.En http://www.rieoei.org/rie26a07.PDF [12-08-2013]

Legislación

Ley Orgánica 8/1985 del Derecho a la Educación (LODE).

Ley Orgánica de Ordenación General del Sistema Educativo (L.O.G.S.E., 1990).

Ley Orgánica de Calidad de la Educación (L.O.C.E., 2002).

Ley Orgánica de Educación (L.O.E., 2006).

\section{Los autores}

Amaro Agudo, Ana. Licenciada en Pedagogía en 1998 y Doctora en Pedagogía por la Universidad de Granada en 2002. Tesis doctoral "El movimiento de ciudades educadoras en España: una investigación evaluativa". Docente como PT en un centro de compensatoria 2004-2009, realizando labores de apoyo a la integración. Profesora Titular del centro de Magisterio la Inmaculada adscrito a la Universidad de Granada desde 2008. Coordinadora del área de Didáctica y Organización escolar durante el curso 2011/2012. Profesora del Máster sobre Educación Inclusiva en la Universidad Católica de Valencia San Vicente Mártir. Campo de investigación sobre educación inclusiva en diferentes proyectos. Profesora investigadora a tiempo parcial en grupo de investigación AREA. Departamento de Didáctica y Organización Escolar. Facultad de Ciencias de la Educación de la Universidad de Granada. Miembro del equipo 
de investigación del Proyecto SECS EVALNEC (SISTEMA EDUCATIVO DE COHESION SOCIAL: DISEÑO DE UN MODELO DE EVALUACION DE NECESIDADES). Ministerio de Economía y Competitividad. Proyecto I+D. (2013-2015).

Navarro Mateu, Diego. Profesor Universitario desde hace más de 15 años y gran número de acciones formativas realizadas en torno al ámbito de la discapacidad y los trastornos del desarrollo efectuados dentro y fuera del entorno universitario. El desempeño de tareas de gobierno y gestión en la universidad, como son las siguientes: Coordinador de estudios de las especialidades de Magisterio Audición y Lenguaje, y de Educación especial, (actual vicedecano), durante 5 cursos académicos. Codirector postgrado La Lengua de SIGNOS EN LA Escuela durante cinco ediciones. Perteneciente al Vicedecanato de Prácticas de la Escuela de Magisterio, desde los últimos tres cursos académicos hasta la actualidad. Colaborador en la puesta en marcha del programa de Acción Tutorial de la Universidad Católica san Vicente Mártir. En la actualidad nombrado Director del Departamento de Psicología y Necesidades Educativas EspecialesC.G.28.05.09 - Acta n ${ }^{\circ} 127$ de la Facultad de Ciencias de la Educación y del Deporte de la Universidad Católica de Valencia San Vicente Mártir. Director del Máster en Educación Inclusiva. Los diferentes trabajos realizados desde la empresa Capacita Consultores, empresa especializada en estudios, formación e investigación sobre el tercer sector, algunos trabajos llevados a cabo son: Guía de Buenas Prácticas y Acciones positivas para la Igualdad de la Mujer en el Trabajo. Diagnóstico e inventario de Recursos de Conciliación de la vida familiar y laboral en la Comarca de la Sabor. Guía práctica para la conciliación de la vida familiar y laboral. Estudio "Oportunidades de negocio para la mujer en Alicante", etc. Estudio "Causas de segregación vertical por razones de género en el mercado laboral Director del Departamento de Calidad de la Federación de Centros Religiosos de la Enseñanza FERE (2006- 2009), Comunidad Valenciana. Impulsando y desarrollando actividades encaminadas a la implantación de sistemas de gestión de calidad en los centros educativos, desarrollo de Jornadas, seminarios, un modelo de reconocimientos basado en el modelo de calidad EFQM, etc. 